\title{
Correction to: Association Between Ov16 Seropositivity and Neurocognitive Performance Among Children in Rural Cameroon: a Pilot Study
}

\author{
Joseph Nelson Siewe Fodjo ${ }^{1,2}$. Wepnyu Y. Njamnshi ${ }^{2,3} \cdot$ Leonard Ngarka ${ }^{2,3,4} \cdot$ Leonard N. Nfor $^{2,3,4} \cdot$ Constance Ayuk $^{3}$. \\ Noelar N. Mundih ${ }^{3} \cdot$ Hilda T. Ekwoge $\mathrm{e}^{6,7} \cdot \mathrm{Kevin} \mathrm{Nganchfu}^{3} \cdot \mathrm{K}$. \\ Pernelle Ngoundjou ${ }^{3}$. Edward Awasume ${ }^{3}$. George Ashu ${ }^{3}$. Earnest N. Tabah ${ }^{3,5,8}$. Robert Colebunders ${ }^{1}$. \\ Alfred K. Njamnshi ${ }^{2,3,4}$
}

Published online: 14 November 2021

(c) The Author(s), under exclusive licence to American Academy of Pediatric Neuropsychology 2021

\section{Correction to: Journal of Pediatric Neuropsychology https://doi.org/10.1007/s40817-021-00111-z}

In the original online publication there were errors in the author affiliations. The original article has been corrected.

Publisher's Note Springer Nature remains neutral with regard to jurisdictional claims in published maps and institutional affiliations.

The original article can be found online at https://doi.org/10.1007/ s40817-021-00111-z.

Alfred K. Njamnshi

alfred.njamnshi@brainafrica.org

1 Global Health Institute, University of Antwerp, Antwerp, Belgium

2 Brain Research Africa Initiative (BRAIN), Yaoundé, Cameroon

3 Neuroscience LabFaculty of Medicine \& Biomedical Sciences, The University of Yaoundé I, Yaoundé, Cameroon

4 Department of Neurology, Yaoundé Central Hospital, Yaoundé, Cameroon

5 Faculty of Medicine \& Pharmaceutical Sciences, The University of Dschang, Dschang, Cameroon

6 Buea Regional Hospital, Buea, Cameroon

7 HILPharma Organization, Yaoundé, Cameroon

8 Ministry of Public Health, Yaoundé, Cameroon 